\title{
Surfaces with Piecewise Linear Support Functions over Spherical Triangulations
}

\author{
Henrik Almegaard ${ }^{a}$, Anne Bagger ${ }^{a}$, Jens Gravesen $^{b}$, \\ Bert Jüttler ${ }^{c}$ and Zbynek Sír ${ }^{d}$ \\ ${ }^{a}$ Technical University of Denmark, Dept. of Civil Engineering/ ${ }^{b}$ of Mathematics \\ ${ }^{c}$ Johannes Kepler University, Institute of Applied Geometry, Linz, Austria \\ ${ }^{d}$ Charles University, Faculty of Mathematics and Physics, Prague, Czech Republic \\ aeb@byg.dtu.dk, hal@byg.dtu.dk, j.gravesen@mat.dtu.dk, \\ bert.juettler@jku.at, sir@karlin.mff.cuni.cz
}

\begin{abstract}
Given a smooth surface patch we construct an approximating piecewise linear structure. More precisely, we produce a mesh for which virtually all vertices have valency three. We present two methods for the construction of meshes whose facets are tangent to the original surface. These two methods can deal with elliptic and hyperbolic surfaces, respectively. In order to describe and to derive the construction, which is based on a projective duality, we use the so-called support function representation of the surface and of the mesh, where the latter one has a piecewise linear support function.
\end{abstract}

\section{Introduction}

Surface patches whose Gauss image (i.e., the mapping induced by the unit normals) defines a bijection between the surface and the co-domain of the Gauss image, which is a certain subset of the unit sphere, can be represented by their support functions. In this representation, a surface is described by the distance of its tangent planes to the origin of the coordinate system, which defines a function on the unit sphere. This representation is a fundamental tool in the field of convex geometry, see e.g. $[2,5,6]$. It has many potential applications in Computer Aided Design, as pointed out by Sabin [14].

Particular classes of support functions correspond to special types of surfaces. The case of surfaces with polynomial support functions has been discussed in [15]. As shown there, these surfaces are obtained by forming the convolution of certain surfaces of revolution whose meridians are special trochoids.

After polynomials, the simplest possible class of support functions are piecewise linear functions, which will be studied in this paper. These functions correspond to piecewise linear surfaces (meshes) with the property that the majority of vertices have valence three, and the majority of the facets are hexagons.

In order to solve the problem of reconstructing a mesh from its planar projections, similar meshes were constructed in [13] with the help of three-dimensional Voronoi diagrams. An optimization-based framework for applications in architecture was presented in [4]. By applying a projective duality to a mesh produced 
by a subdivision surface, such meshes were produced in [9]. The relation between general polyhedra and their dual objects with respect to a projective duality (such as the polarity with respect to the unit sphere, which is sometimes called the "polar reciprocation") has been studied in classical geometry $[3,16,17]$. In particular, polyhedra with special properties, such as uniform polyhedra (where all stars of vertices have the same shape), have been analyzed. It should not be confused with the graph-theoretical duality, which is considered, e.g., in [11].

In this paper we use the support function to describe both the meshes and their dual objects. The support function is somehow "in-between" both objects, and it allows to describe both of them in a simple way. The use of the support functions automatically leads to a restriction of the class of dual meshes; these meshes have to be star-shaped with respect to the origin.

A possible application of the meshes described by piecewise linear support function comes from architecture, where free-form surfaces are often approximated by planar facets [4].

Motivated by applications in architectural design, constructions of quadrangular meshes with planar facets have recently been described in [10]. These meshes can be seen as discretizations of conjugate networks of curves on surfaces. In particular, as a discretization of the network of principal curvature lines, [10] introduces the class of conical (quadrangular) meshes. These meshes are characterized by the property that - for any offset distance - the four planes obtained by offsetting the planes spanned by the four quadrangles sharing a given vertex intersect in a single point. This is a desirable property for architectural design, since it facilitates the construction of offset (or parallel) structures, which may be needed for statical reasons. These meshes have further been analyzed in [12].

In the present paper we focus on meshes with planar faces, where virtually all vertices possess valency 3 (i.e., trihedral meshes). Consequently, most faces are planar hexagons. Clearly, any three planes parallel to the three faces (which are assumed to be mutually different) sharing a given vertex intersect in a single point, and the existence of offset structures is therefore guaranteed. The constructions described below are capable of producing meshes with exactly planar faces, as they work without numerical optimization.

The remainder of this paper is organized as follows. The next section presents some background information about shell structures in architectural design. In particular it discusses the different possible structures with planar facets. Section 3 introduces support functions and discusses their use for describing the polarity with respect to the unit sphere. The special case of piecewise linear support functions is described in Section 4. In particular, it is shown that these surfaces define a star-shaped triangular mesh and a primal mesh with quasiconvex facets, which are not always simple. Finally, in order to approximate a given surface by a mesh corresponding to a piecewise linear support function, the construction of tangent meshes is described in Section 5. In the case of hyperbolic surface patches, this can be achieved with the help of the parameterization by asymptotic lines, while elliptic surface patches can be dealt with via convex hull computations. The methods can be applied to surface patches whose Gauss 
image defines a bijection between the patch and the corresponding spherical domain. In the case of elliptic surface patches, the spherical domain is required to be convex. Finally we conclude the paper.

\section{Shell structures for architectural design}

Shell structures, and in particular their realizations as piecewise linear structures, are attractive tools for architectural design. We summarize the background from statics and discuss the case of faceted glass shells.

\subsection{Shell structures}

Shell structures can in general be considered as structurally efficient structures. The efficiency is due to the fact that the curved form reduces the bending strength needed to carry the loads to almost zero, hence the shell surface can be very thin. For instance, an egg shell can take up considerable load as long as it is unbroken. It can drop from the nest without breaking. Only a concentrated point load - and especially a point load from the inside - will break it. Once it is broken, the egg shell is very weak. This is because the support conditions have changed, so that now only the bending strength is carrying the load.

In principle the bending strength can be zero, if the support conditions are appropriate. Then a specific ideal structural model, the membrane shell model, can be used. In the membrane shell model only membrane forces are considered. Membrane forces are in-plane shear and normal forces, and for a curved surface the considered plane is the tangent plane. Membrane forces are the type of forces that develop in tent structures, but in tents only tension forces can be developed. In membrane shell structures both tension and compression forces are developed. This means in principle that the shell surface should be considered perfectly bendable, both geometrically and statically. In order to keep the surface geometrically and statically determinate, the support conditions along the edge have to fulfill certain rules. Furthermore, the shell design has to follow certain guidelines in order to be stiff and to avoid too large internal stresses [1]. In practice though, shells have to have some bending stiffness in order to carry concentrated loads and to avoid instability from buckling.

Membrane shell structures can be designed either with smooth curved surfaces or with surfaces composed of plane facets - faceted surfaces (Fig. 1). From a construction point of view, the faceted surfaces are very attractive compared to the curved surfaces, as they are less complicated to build. The planar facets make them much easier to describe and produce industrially than doubly curved surfaces. At the same time, various standard materials and components can be used. For faceted surfaces, three geometrically and statically different systems are of interest.

- Triangular facets with six-way vertices. Statically this system generates concentrated forces along the edges, leading to the well known triangulated truss shell structures consisting of bars and joints (Fig. 2, left). 

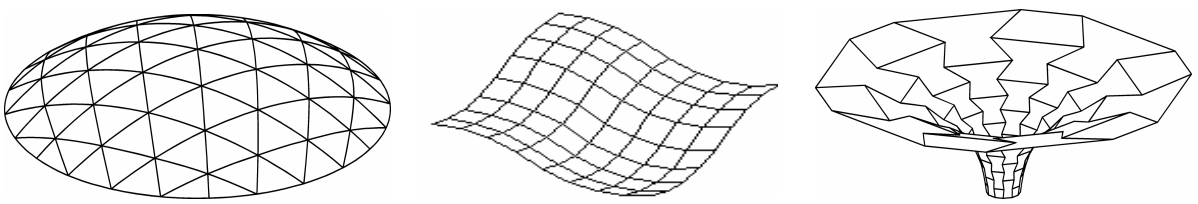

Fig. 1. Faceted shell structures. A) Triangulated truss shell structure. B) Quadrangular hybrid shell structure. C) Three-way vertices plate shell structure.
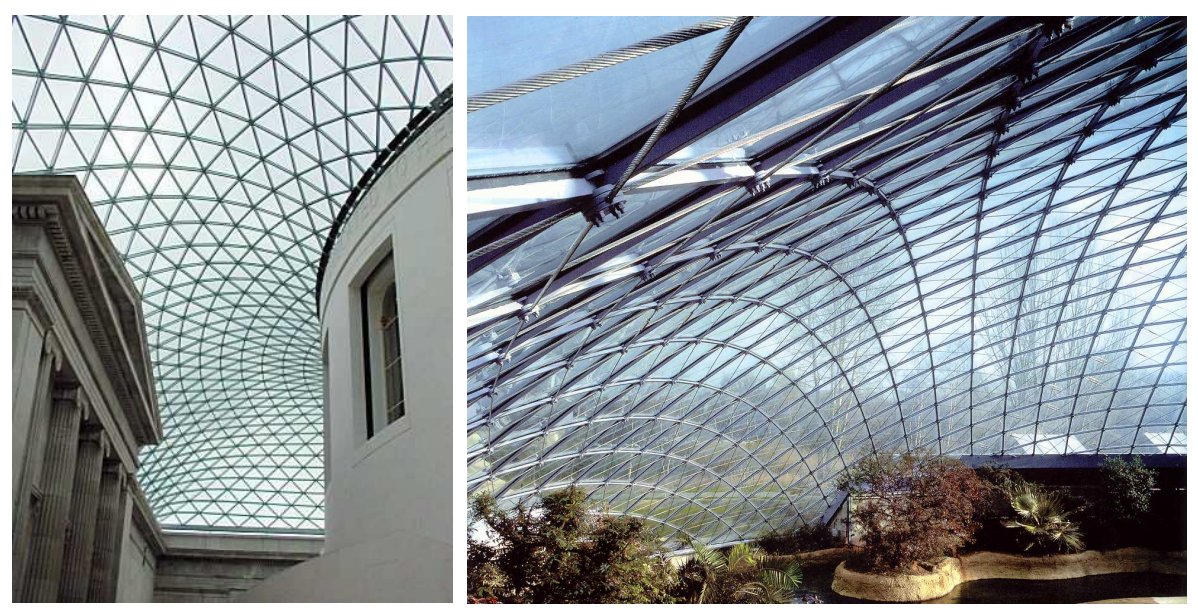

Fig. 2. Left: Triangulated truss shell structure consisting of bars and joints (Great Court, British Museum, London). Right: Hybrid type of structure consisting of a quadrangular truss stabilized by pre-stressed diagonal cables (Hippo House, Berlin Zoo)

- Quadrangular facets with four-way vertices. A widely used example is faceted translation shells (Fig. 2, right). Statically this system generates concentrated forces along the edges and shear forces in the facets, leading to a hybrid type of structure consisting of a quadrangular truss stabilized by plates or pre-stressed diagonal cables.

- Hexagonal facets with three-way vertices. Geometrically this system is dual to the triangulated system. Statically this system only generates in-plane shear and normal plate forces in the facets, leading to plate shell structures (Fig. 3, left).

The structural systems and forces here mentioned are the global structural systems and forces. They are all in-plane forces and equal to the membrane forces in smooth curved shells. This means that if the support conditions are appropriate, no bending stiffness is needed in the connections between the elements. Only locally the out of plane loads applied have to be carried to the edges of the facets by bending forces. 

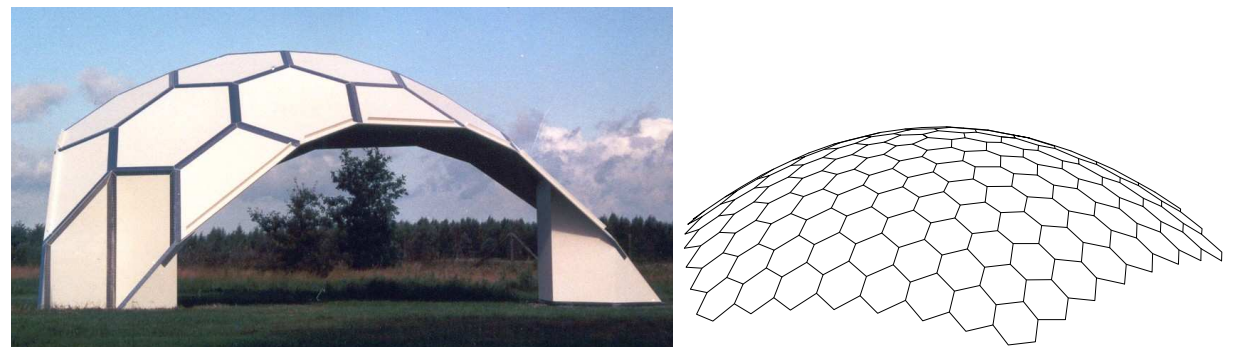

Fig. 3. Left: Plate shell structure consisting of plates primarily subjected to in-plane shear and normal forces (Full scale model, SBI Hørsholm). Right: Principal layout for a load carrying faceted shell structure of glass.

\subsection{Faceted glass shell}

Glass is already to some extent used for load carrying structural members like beams and columns. The structural use of glass is troubled by a brittle behavior of the material, and a limited capacity for carrying tension forces. However, these characteristics can be taken into account in the design process in various ways, thereby opening up for a world of transparent load carrying structures.

The load bearing capacity of glass is similar to that of wood when in tension, and to steel when in compression. In principle, glass is an exceptionally strong material, but in reality small flaws are distributed randomly over the surface. When the allowable tension stress is reached at the glass surface, one of these small flaws (a small crack) eventually will start to open. Since a redistribution of the stresses is not possible in glass, fracture will occur - hence the brittle behavior.

As described earlier, bending stresses are minimized in shell structures, which are appropriately shaped and supported, and the load is transferred primarily via in-plane stresses (membrane stresses). This allows for a better utilization of the capacity of the structural material, since stresses are distributed evenly over the thickness of the structure instead of concentrated at the surfaces. The stiffness to weight ratio of a shell structure - smooth or faceted - is remarkably good, since the absorption of loads is provided by the overall global shape of the structure, and not by a local sectional area. If structural instability can be avoided, the structural thickness of a smooth shell can easily be as little as 1/1000 of the span, or less. This is where glass becomes an interesting possibility as the load carrying material. A span of 15 meters corresponds to a glass thickness of $15 \mathrm{~mm}$, if the thickness/span ratio of $1 / 1000$ is achieved, and that is not an unrealistically large thickness for a laminated glass pane.

In order to avoid the high cost of the production of glass of double curvature, faceted glass shell structures are considered as an alternative to the smooth glass shell. Planar pieces of glass are more easily described, produced and transported. The glass thickness will increase somewhat compared to the smooth shell, since the structure will be burdened by unfavorable local effects. 
A part of a faceted paraboloid of revolution is shown in Fig. 3, right. This geometrical drawing is in the following considered as the principle layout for a load carrying faceted shell structure of glass. The span is about 16 meters, and the size of the facets is roughly 1.2 meters. The glass elements are all planar, and the majority are shaped as hexagons. The central piece of glass is a pentagon, allowing the hexagons to keep roughly the same size, even though the structure is curved. The joints connecting the glass panes are designed to be able to transfer in-plane stresses.

\section{Support function and dual surface}

We use the polarity with respect to the unit sphere to define the dual surface of a given surface and discuss its relation to the support function representation.

\subsection{The polarity with respect to the unit sphere}

Any non-degenerate quadric surface in three-dimensional space defines an associated polarity. In particular, we consider the unit sphere $\mathbb{S}^{2}$ (centered at the origin). The associated polarity $\pi$ maps the point

$$
\mathbf{p}=\left(p_{1}, p_{2}, p_{3}\right)^{\top}
$$

into the plane

$$
\mathbf{P}:\left\{\mathbf{x}=\left(x_{1}, x_{2}, x_{3}\right)^{\top}: 1=\mathbf{p} \cdot \mathbf{x}=p_{1} x_{1}+p_{2} x_{2}+p_{3} x_{3}\right\}
$$

and vice versa, i.e., $\mathbf{P}=\pi(\mathbf{p})$ and $\mathbf{p}=\pi(\mathbf{P})$. It is defined for all points except for the origin, $\mathbf{p} \in \mathbb{R}^{3} \backslash\{\mathbf{0}\}$, and for all planes which do not pass through the origin $^{1}$.

The polarity $\pi$ preserves the incidence of points and planes: if a point $\mathbf{p}$ belongs to a plane $\mathbf{Q}$, then the plane $\pi(\mathbf{p})$ passes through the the point $\pi(\mathbf{Q})$.

The plane $\pi(\mathbf{p})$ is perpendicular to the line $\{\lambda \mathbf{p}: \lambda \in \mathbb{R}\}$. The distances $d$ and $D$ of the point and of its image plane from the origin satisfy $d D=1$. In particular, each point of the unit sphere is mapped to the tangent plane at itself.

\subsection{The dual surface}

We consider a segment of a smooth $\left(\mathcal{C}^{2}\right)$ surface $\mathbf{p}(u, v),(u, v) \in \Omega$. Each point has an associated unit normal

$$
\mathbf{N}: \Omega \rightarrow \mathbb{S}^{2}:(u, v) \mapsto \mathbf{N}(u, v)
$$

\footnotetext{
${ }^{1}$ These restrictions can be avoided by considering the projective closure of the threedimensional space. The origin corresponds to the plane at infinity, and the infinite points correspond to planes passing through the origin.
} 


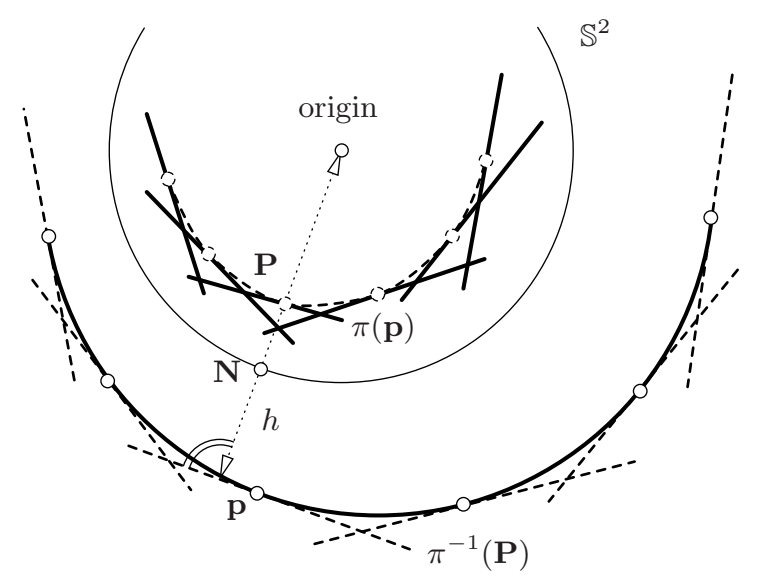

Fig. 4. The polarity $\pi$ maps the points $\mathbf{p}$ of the primal surface to the tangents $\pi(\mathbf{p})$ of the dual surface (both shown as solid lines), and the tangent planes $\pi^{-1}(\mathbf{P})=\pi(\mathbf{P})$ of the primal surface to the points $\mathbf{P}$ of the dual surface (both shown as dashed lines). The figure shows the two-dimensional situation.

which depends smoothly on $u, v$ and defines an orientation of the surface. We assume that the mapping $\mathbf{N}$ is a bijective mapping between $\Omega$ and $\mathbf{N}(\Omega) \subseteq \mathbb{S}^{2}$. This is satisfied, provided that the surface does not contain parabolic or singular points and the domain is sufficiently small.

The polarity $\pi$ is now applied to the points of the surface patch $\mathbf{p}(u, v)$. This produces the two-parameter family of planes

$$
\{\mathbf{x}: 1=\mathbf{x} \cdot \mathbf{p}(u, v)\} \text {. }
$$

At the same time, the polarity is applied to the two-parameter family of tangent planes of the surface patch $\mathbf{p}(u, v)$. This leads to the points

$$
\mathbf{P}(u, v)=\frac{1}{H(u, v)} \mathbf{N}(u, v)
$$

where

$$
H: \Omega \rightarrow \mathbb{R}:(u, v) \mapsto \mathbf{N}(u, v) \cdot \mathbf{p}(u, v)
$$

measures the distance between the tangent plane and the origin.

These points form the dual surface of $\mathbf{p}(u, v)$ with respect to the polarity $\pi$, cf. Fig. 4. Its tangent planes are the images (4) of the points. The dual surface is well-defined provided that $H \neq 0$, i.e., no tangent plane of the surface patch $\mathbf{p}(u, v)$ contains the origin $\mathbf{0}$.

The signs of the Gaussian curvatures of the surface patch $\mathbf{p}(u, v)$ and of its dual surface $\mathbf{P}(u, v)$ are identical. If both surfaces have negative Gaussian curvature, then the asymptotic lines of $\mathbf{p}(u, v)$ correspond to the asymptotic lines of the dual surface. 
Remark 1. Dual curves and surfaces have been considered by Hoschek [8] for detecting sign changes of the curvature and the Gaussian curvature. Hoschek uses the polarity with respect to the imaginary unit sphere, which corresponds to changing the sign of the right-hand side in (5).

\subsection{Support functions of surfaces}

The function

$$
h: \mathbf{N}(\Omega) \rightarrow \mathbb{R}: h=H \circ \mathbf{N}^{-1}
$$

which is obtained by composing the inverse of the map $\mathbf{N}$ defined in (3) with the function $H$ defined in (6), is called the support function of the given surface patch. Support functions are a classical tool in the field of convex geometry [2, $5,6]$. Recently, curves and surfaces with polynomial support functions have been studied by three of the authors [15].

If a support function $h \in \mathcal{C}^{1}(D, \mathbb{R})$ is given, where $D \subseteq \mathbb{S}^{2}$, then the associated surface patch can be constructed by computing the envelope of the planes

$$
\{\mathbf{x}: h(\mathbf{n})=\mathbf{n} \cdot \mathbf{x}\}, \quad \mathbf{n} \in D .
$$

For any $\mathbf{n} \in D$, the corresponding point on the envelope is

$$
\mathbf{p}(\mathbf{n})=h(\mathbf{n}) \mathbf{n}+\left(\nabla_{\mathbb{S}^{2}} h\right)(\mathbf{n})
$$

where $\nabla_{\mathbb{S}^{2}}$ is the embedded intrinsic gradient of the support function $h$ with respect to the unit sphere. If $h^{*} \in \mathcal{C}^{1}\left(\mathbb{R}^{3}, \mathbb{R}\right)$ is a scalar field whose restriction to $\mathbb{S}^{2}$ equals $h$, then

$$
\left(\nabla_{\mathbb{S}^{2}} h\right)(\mathbf{n})=\left(\nabla h^{*}\right)(\mathbf{n})-\left(\left(\nabla h^{*}\right)(\mathbf{n}) \cdot \mathbf{n}\right) \mathbf{n} .
$$

The mapping $\mathbf{n} \mapsto \mathbf{p}(\mathbf{n})$ is the inverse of the Gauss map of the surface patch.

The dual surface can also be obtained from the support function $h$,

$$
\mathbf{P}(\mathbf{n})=\frac{1}{h(\mathbf{n})} \mathbf{n},
$$

cf. (5).

Example 1. We consider the support function $h$ which is obtained by restricting $h^{*}=3+5 n_{1}$ to the unit sphere $\mathbb{S}^{2}$. The intrinsic gradient (10) equals

$$
\left(\nabla_{\mathbb{S}^{2}} h\right)(\mathbf{n})=\left(5-5 n_{1}^{2},-5 n_{1} n_{2},-5 n_{1} n_{3}\right)^{\top} .
$$

Using (9) and (11) we obtain the equations of the primal and the dual surface,

$$
\mathbf{p}(\mathbf{n})=\left(3 n_{1}+5,3 n_{2}, 3 n_{3}\right)^{\top}, \quad \text { and } \quad \mathbf{P}(\mathbf{n})=\frac{1}{3+5 n_{1}}\left(n_{1}, n_{2}, n_{3}\right)^{\top},
$$

respectively. Now one may substitute any parameterization of the unit sphere for $\mathbf{n}=\left(n_{1}, n_{2}, n_{3}\right)^{\top}$ in order to parameterize these two surfaces. In this case as for any linear polynomial $h^{*}$ with non-vanishing constant term - we obtain a sphere and a quadric of revolution. 
Remark 2. The surface defined by (9) is not always regular. If $h \in \mathcal{C}^{2}(D, \mathbb{R})$, then the regularity can be characterized by the intrinsic Hessian of $h$ : If the mapping $\left(\operatorname{Hess}_{\mathbb{S}^{2}}+\mathrm{id}\right) h$ has full rank, then the surface (9) is regular. See [15] for a detailed discussion of regularity.

Remark 3. The two support functions $h$ and $-(\rho \circ h)$, where $\rho$ is the reflection $\rho: \mathbf{n} \mapsto-\mathbf{n}$ with respect to the origin, define the same surface, but with different orientations.

\section{Piecewise Linear Support Functions}

We define piecewise linear functions on the unit sphere and discuss the primal and dual meshes associated with them.

\subsection{Piecewise linear functions on spherical triangulations}

Consider three linearly independent points $\mathbf{v}_{1}, \mathbf{v}_{2}, \mathbf{v}_{3} \in \mathbb{S}^{2}$ which are assumed to lie in one hemisphere of the unit sphere (i.e., there exists a vector $\mathbf{r} \in \mathbb{R}^{3}$ such that $\left.\mathbf{r} \cdot \mathbf{v}_{i}>0, i=1,2,3\right)$. The three great circular arcs connecting them, which are contained in the same hemisphere, bound a spherical triangle.

We consider a subset $D \subseteq \mathbb{S}^{2}$ which is bounded by a sequence of great circular arcs with vertices $\mathbf{n}_{1}, \ldots, \mathbf{n}_{k}$. In addition, let $\mathbf{n}_{k+1}, \ldots, \mathbf{n}_{m} \in \operatorname{int} D$ be additional points in the interior of $D$. A spherical triangulation $\mathcal{T}$ of $D$ with vertices $\left(\mathbf{n}_{i}\right)_{i=1, \ldots, m}$ is a collection of spherical triangles satisfying the following three properties:

1. The interiors of any two spherical triangles are disjoint,

2. the intersection of any triangle with the set of vertices consists of exactly three points, which are the vertices of that triangle, and

3. the union of all triangles is equal to $D$.

Now we consider a spherical triangulation $\mathcal{T}$ with vertices $\left(\mathbf{n}_{i}\right)_{i=1, \ldots, m}$. We assume that each vertex $\mathbf{n}_{i}$ is equipped with an associated scalar value $h_{i}$. For any spherical triangle $\triangle_{i j k} \in \mathcal{T}$ with vertices $\mathbf{n}_{i}, \mathbf{n}_{j}, \mathbf{n}_{k}$, we consider the unique homogeneous linear function

$$
h_{i j k}^{*}: \mathbb{R}^{3} \rightarrow \mathbb{R}: \mathbf{x} \mapsto\left(h_{i}, h_{j}, h_{k}\right)\left(\mathbf{n}_{i}, \mathbf{n}_{j}, \mathbf{n}_{k}\right)^{-1} \mathbf{x},
$$

where $\left(\mathbf{n}_{i}, \mathbf{n}_{j}, \mathbf{n}_{k}\right)$ is the $3 \times 3$-matrix with columns $\mathbf{n}_{i}, \mathbf{n}_{j}$ and $\mathbf{n}_{k}$, and restrict it to $\triangle_{i j k}$. This function satisfies $h_{i j k}^{*}\left(\mathbf{n}_{l}\right)=h_{l}$ for $l \in\{i, j, k\}$. The collection of all these functions defines a unique piecewise linear function $h \in \mathcal{C}(D, \mathbb{R})$ over the spherical triangulation which interpolates the given values,

$$
h\left(\mathbf{n}_{i}\right)=h_{i}, \quad i=1, \ldots, m .
$$

In the remainder of this paper we assume that all $h_{i}$ are positive,

$$
h_{i}>0, \quad i=1, \ldots, m .
$$

The piecewise linear interpolant $h$ is then also positive for all $\mathbf{x} \in D$. 
Remark 4. Index triples $i j k$ as for $\triangle_{i j k}$ with the same set of indices, but different order, will be identified, i.e. $\triangle_{i j k}=\triangle_{i k j}=\triangle_{j i k}$ etc. That is, $i j k$ represents a subset of $\{1, \ldots, m\}$ with cardinality three, and these subsets are used to label the triangles, etc.

\subsection{The dual mesh}

With the help of the relationship between the dual surface and the support function, we now define the dual mesh via (11). More precisely, for each spherical triangle $\triangle_{i j k} \in \mathcal{T}$ with vertices $\mathbf{n}_{i}, \mathbf{n}_{j}, \mathbf{n}_{k}$ we obtain a segment of the dual mesh,

$$
\mathbf{P}_{i j k}(\mathbf{n})=\frac{1}{h_{i j k}^{*}(\mathbf{n})} \mathbf{n}, \quad \mathbf{n} \in \triangle_{i j k} .
$$

Using the parameterization

$$
\mathbf{n}(u, v, w)=\frac{u \mathbf{n}_{i}+v \mathbf{n}_{j}+w \mathbf{n}_{k}}{\left\|u \mathbf{n}_{i}+v \mathbf{n}_{j}+w \mathbf{n}_{k}\right\|}, \quad u+v+w=1 ; u, v, w \geq 0
$$

of the spherical triangle and by exploiting the fact that $h_{i j k}^{*}$ is homogeneous, one gets the rational linear parameterization

$$
\mathbf{P}_{i j k}(\mathbf{n}(u, v, w))=\frac{u \mathbf{n}_{i}+v \mathbf{n}_{j}+w \mathbf{n}_{k}}{\left(h_{i}, h_{j}, h_{k}\right)\left(\mathbf{n}_{i}, \mathbf{n}_{j}, \mathbf{n}_{k}\right)^{-1}\left(u \mathbf{n}_{i}+v \mathbf{n}_{j}+w \mathbf{n}_{k}\right)}
$$

which describes the triangle with vertices

$$
\mathbf{t}_{l}=\frac{1}{h_{l}} \mathbf{n}_{l}, \quad l \in\{i, j, k\} .
$$

This leads to

Proposition 1. The dual mesh $\mathbf{P}$ associated with the piecewise linear support function $h \in \mathcal{C}(D, \mathbb{R})$ satisfying (15) over the given spherical triangulation $\mathcal{T}$ of $D$ with vertices $\left(\mathbf{n}_{i}\right)_{i=1, \ldots, m}$ is the triangular mesh with vertices $\left(\mathbf{t}_{i}\right)_{i=1, \ldots, m}$, which has the same connectivity as $\mathcal{T}$. This mesh is star-shaped with respect to the origin; each ray $\lambda \mathbf{n}$ with $\lambda \geq 0, \mathbf{n} \in D$ intersects the mesh $\mathbf{P}$ in a single point.

\subsection{Quasi-convex polygons}

Before discussing the primal mesh, we consider planar curve-like objects with piecewise linear support functions.

Definition 1. Consider a closed polygon with $v$ vertices $\left(\mathbf{q}_{i}\right)_{i=0, \ldots, v-1}$ which lies in a plane $\mathbf{T} \subset \mathbb{R}^{3}$, where any triple of neighboring points is assumed to be non-collinear. For each edge

$$
E_{i}=\left\{(1-t) \mathbf{q}_{i}+t \mathbf{q}_{i+1 \bmod v}: t \in[0,1]\right\}, \quad i=0, \ldots, v-1,
$$




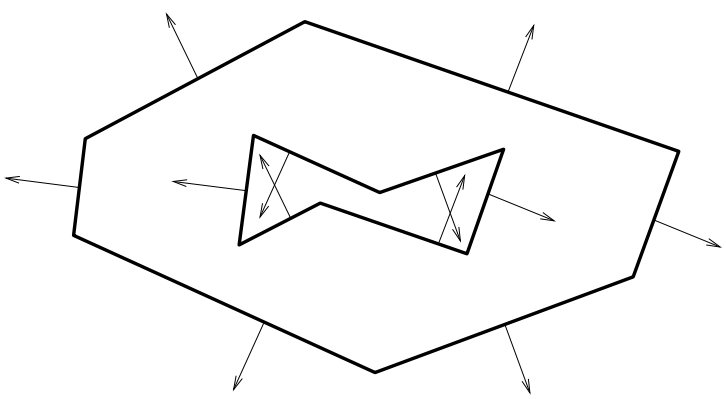

Fig. 5. Quasi-convex polygons as offsets of convex polygons.

we choose one of the two possible unit normal vectors $\mathbf{n}_{i}$ lying in $\mathbf{T}$. The polygon along with the unit normals is then called an oriented polygon. At each vertex $\mathbf{q}_{i}$ we consider the convex cone

$$
C_{i}=\left\{\lambda \mathbf{n}_{i-1}+\mu \mathbf{n}_{i \bmod v}: \lambda \geq 0, \mu>0\right\}, \quad i=1, \ldots, v .
$$

The oriented polygon is said to be quasi-convex if $i \neq j$ implies $C_{i} \cap C_{j}=\emptyset$.

According to this definition, a quasi-convex polygon is characterized by the fact that the oriented unit normals trace the unit circle exactly once. Any convex polygon, where all edges are oriented by choosing either outward or inward pointing normals, is also quasi-convex. A general quasi-convex polygon is obtained as an offset of a convex one, where all edges are simply translated by a certain signed distance, according to the given normals.

Remark 5. If $\mathbf{T}$ is the plane $\mathbb{R}^{2}$, then an oriented polygon can be described by a piecewise linear support function on the unit circle $\mathcal{S}^{1}$. The edges and vertices of the polygon correspond to the nodes and to the linear pieces of this function, respectively.

\subsection{The primal mesh}

Each triangle $\triangle_{i j k} \in \mathcal{T}$ has an associated linear support function $h_{i j k}^{*}$ which defines a triangular facet $\mathbf{P}_{i j k}$ of the dual mesh. By applying the polarity to the plane spanned by this facet, we obtain the corresponding point

$$
\mathbf{p}_{i j k}=\left(\left(h_{i}, h_{j}, h_{k}\right)\left(\mathbf{n}_{i}, \mathbf{n}_{j}, \mathbf{n}_{k}\right)^{-1}\right)^{\top}
$$

of the primal mesh.

For any inner vertex $\mathbf{n}_{i} \in$ int $D$ of the spherical triangulation we consider the star of this vertex, i.e., the set of triangles sharing this vertex,

$$
\mathcal{S}_{i}=\left\{\triangle_{i j k} \in \mathcal{T}: j, k \in\{1, \ldots, m\}\right\} .
$$



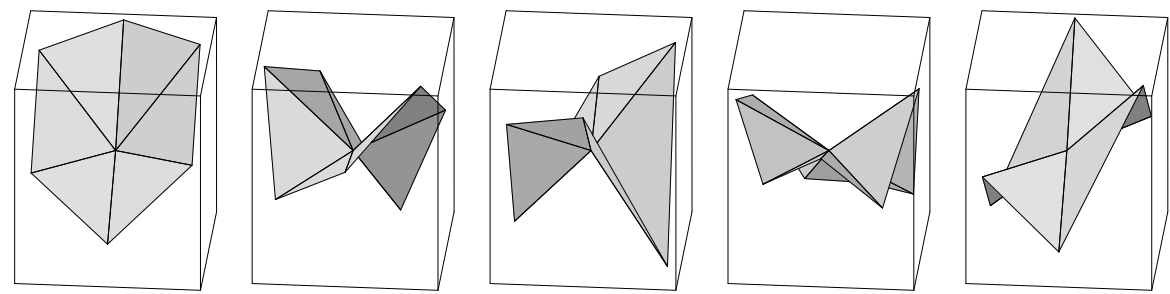

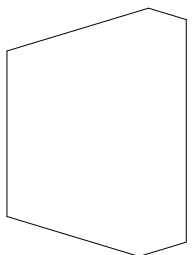

(a)

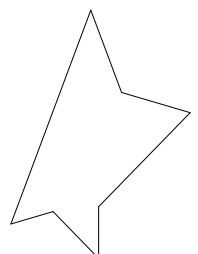

(b)

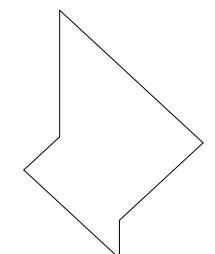

(c)

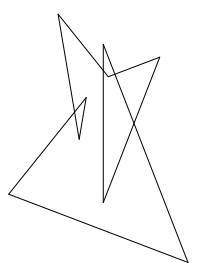

(d)

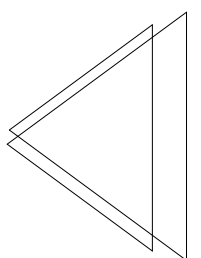

(e)

Fig. 6. Stars of vertices of the dual mesh and the associated quasi-convex polygons of the primal mesh: convex, simple (a); non-convex, simple (b,c); non-convex, non-simple (d,e).

Each triangle $\triangle_{i j k}$ corresponds to a point $\mathbf{p}_{i j k}$. All these points lie in the plane

$$
\mathbf{T}_{i}=\left\{\mathbf{x} \in \mathbb{R}^{3}: \mathbf{x} \cdot \mathbf{n}_{i}=h_{i}\right\},
$$

which is obtained by applying the polarity to the vertex $\mathbf{p}_{i}$. By connecting points, where the corresponding triangles have a common edge, we obtain a polygon.

Example 2. Five different stars and the associated planar polygons are shown in Fig. 6. If the vertex is convex, then the associated polygon is also convex. Otherwise, non-convex and even non-simple polygons may be obtained.

Proposition 2. The primal mesh $\mathbf{p}$ associated with the piecewise linear support function $h \in \mathcal{C}(D, \mathbb{R})$ satisfying (15) over the given spherical triangulation $\mathcal{T}$ of $D$ with vertices $\left(\mathbf{n}_{i}\right)_{i=1, \ldots, m}$ is the set of (not-necessarily simple) quasi-convex planar polygons with vertices $\mathbf{p}_{i j k}$. These polygons will be called the facets of $\mathbf{p}$. For each inner vertex $\mathbf{n}_{i}$ of the triangulation, the star of this vertex defines one of these polygons, and this polygon lies in the plane $\mathbf{T}_{i}$.

Proof. Without loss of generality we consider the star of the vertex $\mathbf{n}_{i}=(0,0,1)^{\top}$. We consider a certain neighborhood of the corresponding vertex $\mathbf{t}_{i}=\left(1 / h_{i}\right) \mathbf{n}_{i}$ of the dual mesh. Since it is star-shaped with respect to the origin, the dual mesh can be represented as

$$
(1-t)\left(\begin{array}{c}
0 \\
0 \\
1 / h_{i}
\end{array}\right)+t\left(\begin{array}{c}
\cos \phi \\
\sin \phi \\
z(\phi)
\end{array}\right), \quad \phi \in \mathbb{R}, t \in[0, \epsilon]
$$

where the $2 \pi$-periodic continuous function $z(\phi)$ is piecewise smooth (in the interiors of the triangles $\mathbf{P}_{i j k}$ ). It has jumps in the first derivatives exactly in those 
directions that correspond to edges shared by neighboring triangles of the dual mesh.

On the one hand, by applying the polarity to the planes spanned by the triangles $\mathbf{P}_{i j k}$ we obtain the vertices of the quasi-convex polygon in the plane $\mathbf{T}_{i}$. On the other hand, by applying the polarity to the parameter lines $\phi=$ constant in (26) we obtain oriented lines with the normal vector $(\cos \phi, \sin \phi, 0)^{\top}$ in this plane. In particular, the lines which correspond to shared edges of neighboring triangular facets of the dual mesh correspond to the edges of the quasi-convex polygon. The remaining lines support that polygon at its vertices.

A simple polygon of the primal mesh will be said to be regular. A primal mesh with only simple polygons will also be said to be regular. We discuss criteria which guarantee regularity.

Lemma 1. Let $\mathbf{t}_{i}$ be a an inner v-vertex of the dual mesh $\mathbf{P}$ and $\mathbf{t}_{j(1)}, \ldots, \mathbf{t}_{j(v)}$ be the neighboring vertices in counterclockwise order. We assume that no two edges meeting at $\mathbf{t}_{i}$ are linearly dependent.

The quasi-convex polygon which corresponds to the star of $\mathbf{t}_{i}$ has a selfintersection if and only if there exists two points $\mathbf{t}_{j(k)}, \mathbf{t}_{j(l)}$ such that the two

points $\mathbf{t}_{j(k-1)}, \mathbf{t}_{j(k+1)}$ are on the same side of the plane spanned by $\mathbf{t}_{j(k)}, \mathbf{t}_{j(l)}$ and $\mathbf{t}_{i}$, and also the two points $\mathbf{t}_{j(l-1)}, \mathbf{t}_{j(l+1)}$ are on the same side of this plane.

Proof. Applying the polarity to the plane spanned by $\mathbf{t}_{j(k)}, \mathbf{t}_{j(l)}$ and $\mathbf{t}_{i}$ gives the intersection point of the polygon.

In particular, if the dual mesh is convex, then all facets of the primal mesh are also convex and therefore regular.

\subsection{Examples}

We use spherical triangulations whose vertices are obtained by applying uniform refinement steps (where each triangle is split into four triangles by halving the edges) to a icosahedron, and projection onto the unit sphere after each refinement step. This gives dual meshes which are adapted to the curvature of the mesh; more facets are used in regions with higher curvature.

Example 3. We consider an ellipsoid with three different diameters and its dual surface, which is again an ellipsoid. The vertices $\mathbf{n}_{i}$ of the spherical triangulation were obtained by applying two steps of refinement to a regular icosahedron. The three symmetry planes of the ellipsoid were aligned with symmetry planes of the icosahedron.

We considered only the upper half of the ellipsoid. The piecewise linear interpolant to the support function of the ellipsoid defines a dual mesh with 90 vertices. This dual mesh is convex, hence the 90 facets of the associated primal mesh do not have any self-intersections. A physical model of this mesh is shown in Figure 7. 

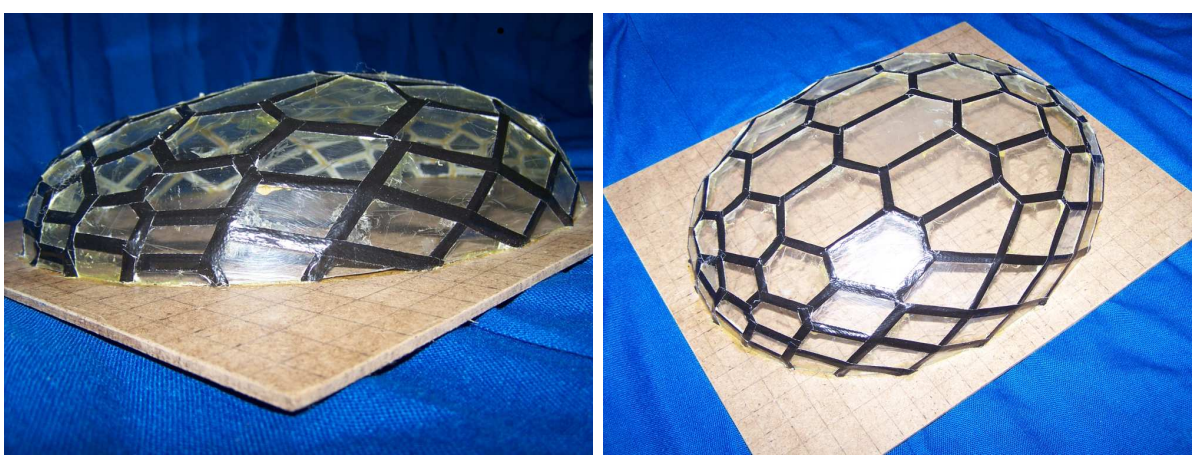

Fig. 7. Example 3. Convex mesh with piecewise linear support function which approximates a segment of an ellipsoid. The mesh consists of 90 facets.

Most facets of the primal mesh have 6 vertices, and most vertices of the primal mesh have valency 3 . In some cases, the edges of the facets are very short, and two of these vertices are virtually identical, leading to a vertex with valency 4 . This situation corresponds to two neighboring triangles of the dual mesh which are almost coplanar.

Example 4. We consider a one-sheeted hyperboloid with three different diameters and its dual surface, which is again a one-sheeted hyperboloid. Similarly to the previous example. the vertices $\mathbf{n}_{i}$ of the spherical triangulation were obtained by applying three steps of refinement (where each triangle is split into four triangles by halving the edges) to a regular icosahedron. The three symmetry planes of the hyperboloid were aligned with symmetry planes of the icosahedron.

We considered a segment of the upper half of the hyperboloid, which is bounded by two vertical planes. The piecewise linear interpolant to the support function of the hyperboloid defines a dual mesh with 169 vertices. This dual mesh is non-convex, but nevertheless each vertex defines a regular facet of the primal mesh. A physical model of this mesh is shown in Figure 8.

As in the previous example, most of the 169 facets of the primal mesh have 6 vertices, and most vertices of the primal mesh have valency 3 .

\section{Tangent meshes}

We discuss the construction of meshes whose facets lie in the tangent planes of a given surface. For a spherical triangulation with vertices $\mathbf{n}_{1}, \ldots, \mathbf{n}_{k}$ and a given (smooth) surface with support function $h(\mathbf{n})$, one can construct piecewise linear support function by choosing $h_{i}=h\left(\mathbf{n}_{i}\right)$ in (14), i.e., by sampling values of the support function of the given surface. However, we have to ensure the regularity of the corresponding primal mesh. 

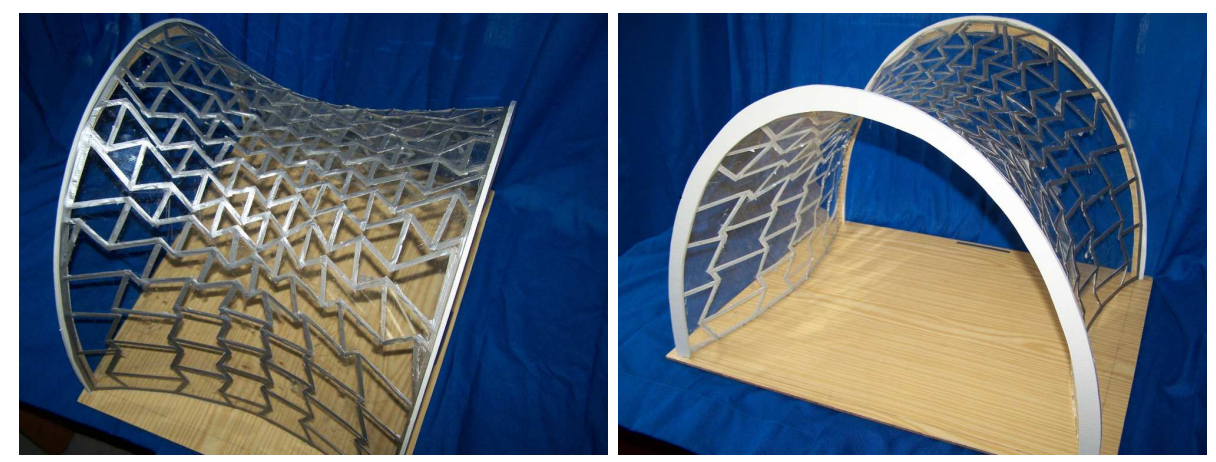

Fig. 8. Example 4. Non-convex mesh with piecewise linear support function which approximates a segment of a one-sheeted hyperboloid. The mesh has 169 facets.

Two approaches will be presented. In the first approach, we consider a sequence of spherical triangulations with decreasing size of the triangles. They correspond to triangular (dual) meshes approximating the dual surface with increasing level of accuracy. Here we analyze the limit shapes of the facets of the primal mesh. This approach is particularly well suited for primal surfaces with only hyperbolic points, see Sections 5.1 and 5.2.

The second approach is restricted to primal surfaces with only elliptic points. In this case a suitable dual mesh can be found via convex hull computation, see Section 5.3.

\subsection{Asymptotic behavior of the vertices of the primal mesh}

We assume that we can generate an increasingly fine triangulation (the dual mesh) of the dual surface, which depends on some step-size $h$. In order to study the limit shape of the facets of the corresponding primal mesh when $h$ tends to zero, we consider the following situation.

We consider the dual surface $\mathbf{P}$ in the vicinity of one of its points $\mathbf{P}_{0}=$ $\mathbf{P}\left(u_{0}, v_{0}\right)$. Consider two $\mathcal{C}^{3}$ curve segments $\mathbf{a}(h), \mathbf{b}(h), h \in(-\epsilon, \epsilon)$, which lie on $\mathbf{P}$ and satisfy $\mathbf{a}(0)=\mathbf{b}(0)=\mathbf{P}_{0}$ and $\mathbf{a}^{\prime} \times \mathbf{b}^{\prime}=\lambda \mathbf{n}_{0}, \lambda>0$, where $\mathbf{n}_{0}$ is the normal of the dual surface at $\mathbf{P}_{0}$ and the prime ' denotes the first derivative with respect to $h$ at $h=0$. Let $\mathbf{Q}(h)$ be the plane spanned by $\mathbf{P}_{0}, \mathbf{a}(h), \mathbf{b}(h)$ and $\mathbf{q}(h)$ its image under the polarity with respect to the unit sphere tangent to $\mathbf{P}$ at $\mathbf{P}_{0}$ and having $\mathbf{n}_{0}$ for outer normal.

After a suitable translation, the sphere can be chosen as the unit sphere $\mathbb{S}^{2}$ centered at the origin, as described in Section 3.1. The plane $\mathbf{Q}(h)$ is well-defined for all $h \neq 0$, provided that $\epsilon$ is sufficiently small.

The limit behavior of $\mathbf{q}(h)$ for decreasing step-size $h$ is described in the following result. 
Lemma 2. The point $\mathbf{q}(h)$ lies in the tangent plane to $\mathbf{P}$ at $\mathbf{P}_{0}$. It satisfies

$$
\lim _{h \rightarrow 0} \mathbf{q}(h)=\mathbf{P}_{0} \quad \text { and } \quad \mathbf{q}^{\prime}=\frac{\left|\mathbf{b}^{\prime}\right|^{2} \kappa_{\mathbf{b}} \mathbf{a}^{\prime}-\left|\mathbf{a}^{\prime}\right|^{2} \kappa_{\mathbf{a}} \mathbf{b}^{\prime}}{2\left(\mathbf{a}^{\prime} \times \mathbf{b}^{\prime}\right) \cdot \mathbf{n}_{0}} \times \mathbf{n}_{0},
$$

where $\kappa_{\mathbf{a}}$ and $\kappa_{\mathbf{b}}$ are normal curvatures of the tangent directions $\mathbf{a}^{\prime}$ and $\mathbf{b}^{\prime}$, respectively.

Proof. The two given curves have Taylor expansions of the form

$$
\begin{aligned}
& \mathbf{a}(h)=\mathbf{P}_{0}+\mathbf{a}^{\prime} h+\frac{1}{2}\left(\left|\mathbf{a}^{\prime}\right|^{2} \kappa_{\mathbf{a}} \mathbf{n}_{0}+\mathbf{t}_{\mathbf{a}}\right) h^{2}+\mathcal{O}\left(h^{3}\right) \\
& \mathbf{b}(h)=\mathbf{P}_{0}+\mathbf{b}^{\prime} h+\frac{1}{2}\left(\left|\mathbf{b}^{\prime}\right|^{2} \kappa_{\mathbf{b}} \mathbf{n}_{0}+\mathbf{t}_{\mathbf{b}}\right) h^{2}+\mathcal{O}\left(h^{3}\right),
\end{aligned}
$$

where the vectors $\mathbf{t}_{\mathbf{a}}, \mathbf{t}_{\mathbf{a}}$ are perpendicular to $\mathbf{n}_{0}$. We compute a normal to the plane $\mathbf{Q}(h)$

$$
\begin{aligned}
\mathbf{N}_{\mathbf{Q}}(h) & =\left(\mathbf{a}(h)-\mathbf{P}_{0}\right) \times\left(\mathbf{b}(h)-\mathbf{P}_{0}\right)= \\
& =\left(\mathbf{a}^{\prime} \times \mathbf{b}^{\prime}\right)\left[h^{2}+c h^{3}\right]+\frac{1}{2}\left(\left|\mathbf{b}^{\prime}\right|^{2} \kappa_{\mathbf{b}} \mathbf{a}^{\prime}-\left|\mathbf{a}^{\prime}\right|^{2} \kappa_{\mathbf{a}} \mathbf{b}^{\prime}\right) \times \mathbf{n}_{0} h^{3}+\mathcal{O}\left(h^{4}\right),
\end{aligned}
$$

where

$$
c=\frac{\left(\mathbf{a}^{\prime} \times \mathbf{t}_{b}-\mathbf{b}^{\prime} \times \mathbf{t}_{a}\right) \cdot\left(\mathbf{a}^{\prime} \times \mathbf{b}^{\prime}\right)}{2\left|\mathbf{a}^{\prime} \times \mathbf{b}^{\prime}\right|^{2}} .
$$

Since the plane $\mathbf{Q}(h)$ contains the point $\mathbf{P}_{0}=\mathbf{n}_{0}$, it has the equation

$$
\mathbf{x} \cdot \mathbf{N}_{\mathbf{Q}}(h)=\mathbf{n}_{0} \cdot \mathbf{N}_{\mathbf{Q}}(h)=\left(\mathbf{a}^{\prime} \times \mathbf{b}^{\prime}\right) \cdot \mathbf{n}_{0}\left[h^{2}+c h^{3}\right]+O\left(h^{4}\right) .
$$

Hence, the corresponding dual point is

$$
\begin{aligned}
\mathbf{q}(h) & =\frac{\mathbf{N}_{\mathbf{Q}}(h)}{\left(\mathbf{a}^{\prime} \times \mathbf{b}^{\prime}\right) \cdot \mathbf{n}_{0}\left[h^{2}+c h^{3}\right]+\mathcal{O}\left(h^{4}\right)}= \\
& =\mathbf{P}_{0}+\frac{\left|\mathbf{b}^{\prime}\right|^{2} \kappa_{\mathbf{b}} \mathbf{a}^{\prime}-\left|\mathbf{a}^{\prime}\right|^{2} \kappa_{\mathbf{a}} \mathbf{b}^{\prime}}{2\left(\mathbf{a}^{\prime} \times \mathbf{b}^{\prime}\right) \cdot \mathbf{n}_{0}} \times \mathbf{n}_{0} h+\mathcal{O}\left(h^{2}\right) .
\end{aligned}
$$

Though the calculation of $\mathbf{q}(h)$ is not valid for $h=0$, the function $\mathbf{q}$ can be extended to a $\mathcal{C}^{2}$ function by letting $\mathbf{q}(0)=\mathbf{P}_{0}$.

Using a regular parameterization $\mathbf{P}=\mathbf{P}(u, v)$ of the dual surface, we can express $\mathbf{q}^{\prime}$ with the help of the second fundamental form.

Lemma 3. Assume that $\mathbf{P}_{u}\left(u_{0}, v_{0}\right) \times \mathbf{P}_{v}\left(u_{0}, v_{0}\right)$ is a positive multiple of $\mathbf{n}_{0}$, where $\mathbf{P}_{u}, \mathbf{P}_{v}$ denote the first derivatives of $\mathbf{P}$ with respect to the parameters. If $\mathbf{P}_{0}=\mathbf{P}\left(u_{0}, v_{0}\right), \mathbf{a}(h)=\mathbf{P}\left(u_{a}(h), v_{a}(h)\right)$ and $\mathbf{b}(h)=\mathbf{P}\left(u_{b}(h), v_{b}(h)\right)$ then

$$
\begin{aligned}
\mathbf{q}^{\prime} & =\mathbf{P}_{u}^{\perp} \frac{L\left(u_{a}^{\prime} u_{b}^{\prime 2}-u_{a}^{\prime 2} u_{b}^{\prime}\right)+2 M u_{b}^{\prime} u_{a}^{\prime}\left(v_{b}^{\prime}-v_{a}^{\prime}\right)+N\left(u_{a}^{\prime} v_{b}^{\prime 2}-v_{a}^{\prime 2} u_{b}^{\prime}\right)}{2\left(u_{a}^{\prime} v_{b}^{\prime}-v_{a}^{\prime} u_{b}^{\prime}\right)}+ \\
& +\mathbf{P}_{v}^{\perp} \frac{L\left(v_{a}^{\prime} u_{b}^{\prime 2}-u_{a}^{\prime 2} v_{b}^{\prime}\right)+2 M v_{a}^{\prime} v_{b}^{\prime}\left(u_{b}^{\prime}-u_{a}^{\prime}\right)+N\left(v_{b}^{\prime 2} v_{a}^{\prime}-v_{b}^{\prime} v_{a}^{\prime 2}\right)}{2\left(u_{a}^{\prime} v_{b}^{\prime}-v_{a}^{\prime} u_{b}^{\prime}\right)}
\end{aligned}
$$


where $L, M, N$ are the coefficients of the second fundamental form at $\mathbf{P}_{0}$, and

$$
\mathbf{P}_{u}^{\perp}=\left.\frac{1}{\sqrt{E F-G^{2}}} \frac{\partial \mathbf{P}}{d u}\right|_{u_{0}, v_{0}} \times \mathbf{n}_{0}, \quad \mathbf{P}_{v}^{\perp}=\left.\frac{1}{\sqrt{E F-G^{2}}} \frac{\partial \mathbf{P}}{d v}\right|_{u_{0}, v_{0}} \times \mathbf{n}_{0},
$$

where $E, F, G$ are the coefficients of the first fundamental form at $\mathbf{P}_{0}$.

This fact can now be verified by a direct computation.

\subsection{Asymptotic behavior of the facets of the dual mesh}

Eq. (34) gives the leading term of $\mathbf{q}(h)$ with respect to the basis $\mathbf{P}_{u}^{\perp}, \mathbf{P}_{v}^{\perp}$. We use it to define the limit shape of a facet.

Definition 2. Consider the dual surface $\mathbf{P}(u, v)$, which is assumed to be regular and $\mathcal{C}^{3}$ in the vicinity of $\mathbf{P}(0,0)$. Let $\left\{\left(u_{1}, v_{2}\right),\left(u_{2}, v_{2}\right), \ldots,\left(u_{n}, v_{n}\right)\right\}$ be the parameter values of the star of the vertex $\mathbf{P}(0,0)$. We define the vertices of the limit shape of the primal facet by applying the expression (34) to the consecutive pairs of dual vertices. More precisely, the limit position of the vertex of the primal facet associated with the triangle with vertices

$$
\mathbf{P}\left(u_{i}, v_{i}\right), \quad \mathbf{P}\left(u_{(i \bmod n)+1}, v_{(i \bmod n)+1}\right), \quad \mathbf{P}(0,0), \quad i=1, \ldots, n,
$$

is found by substituting

$$
u_{a}^{\prime}=u_{i}, \quad v_{a}^{\prime}=v_{i}, \quad u_{b}^{\prime}=u_{(i \bmod n)+1}, \quad \text { and } \quad v_{b}^{\prime}=v_{(i \bmod n)+1}
$$

in the right-hand side of (34), and the limit shape is obtained by connecting consecutive pairs of limit vertices.

The geometrical meaning of the limit shape of the primal polygon is described in the following result.

Proposition 3. We consider the primal facet associated with the star of the dual mesh with apex $\mathbf{P}(0,0)$ and vertices $\mathbf{P}\left(h u_{i}, h v_{i}\right), i=1, \ldots, n$. As $h \rightarrow 0$, the shape of the primal facet tends to the corresponding limit shape.

The proof is a direct consequence of Lemma 3.

By using a regular triangular mesh with edge-length $h$ in the parameter domain of the dual surface, one might expect to obtain regular facets of the

primal mesh as $h$ tends to zero. However, this is not the case as shown by the following example.

Example 5. Consider the following elliptic dual surface

$$
\left(u, v, 1.34 u^{2}+1.89 u v+0.72 v^{2}\right),
$$

and choose the parameters $\left(u_{i}, v_{i}\right)$ as the vertices of a regular hexagon in the $u, v$-plane inscribed in the unit circle. Figure 9 shows the regular polygon (thin) with its limit primal polygon (thick), which is not simple.

In order to obtain a regular primal mesh approximating a patch of of a hyperbolic surface, we propose the following 


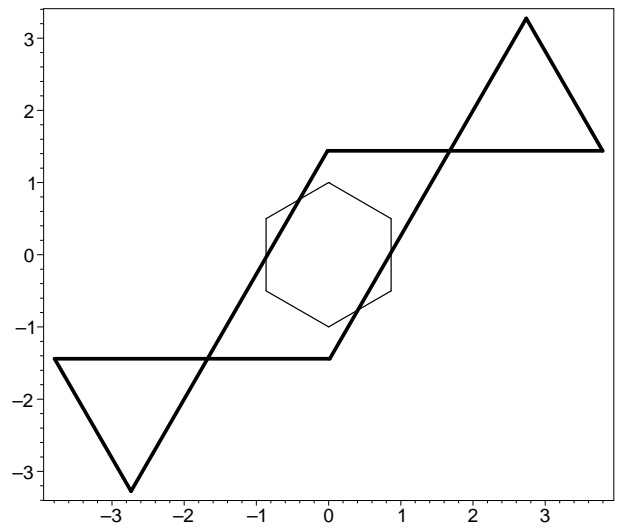

Fig. 9. The limit shape (thick polygon) associated with the star defined by the regular hexagon for the surface (38).

\begin{abstract}
Algorithm 1
Input: Smooth dual hyperbolic surface $\mathbf{P}$ with a $\mathcal{C}^{3}$ curve $\alpha(t)$ lying on it, and step size $h$. The curve $\alpha(t)$ must not touch the asymptotic lines of the dual surface.

Output: Triangular (dual) mesh of the dual surface and associated primal mesh.
\end{abstract}

1. Compute the diagonal points $[n, n]:=\alpha(n t)$.

2. Compute the general grid points $[m, n]$ defined as intersection of the 'vertical' asymptotic line through $[m, m]$ and the 'horizontal' asymptotic line through $[n, n]$. Here, the notions 'vertical' and 'horizontal' are used to distinguish between the two different families of asymptotic lines on the dual surface.

3. Produce the triangular mesh by applying the pattern shown in Figure 10, left.

4. Compute the primal mesh.

Now we can prove that this algorithm produces a sensible output, at least as $h$ tends to zero.

Theorem 1. If the step size $h$ is sufficiently small, then the algorithm produces a regular primal mesh.

Proof. There exists a unique parameterization $\mathbf{P}(u, v)$ of the dual surface such that the the parametric directions are the asymptotic lines and $\alpha(t)=\mathbf{P}(t, t)$. In this parameterization, the second fundamental form satisfies $L=N=0$ and we can compute the limit shape of the primal facets associated with the stars of the dual mesh (indicated by the grey hexagons in Fig. 10, left). In this parameterization we apply directly the formula (34). Note that $L=N=0$. Setting $M=1$ we obtain the regular limit shape shown in Figure 10, right.

Different values of $M$ only scale the limit shape. Note that this shape corresponds to the polarity with respect to a unit sphere which is tangent to the dual 

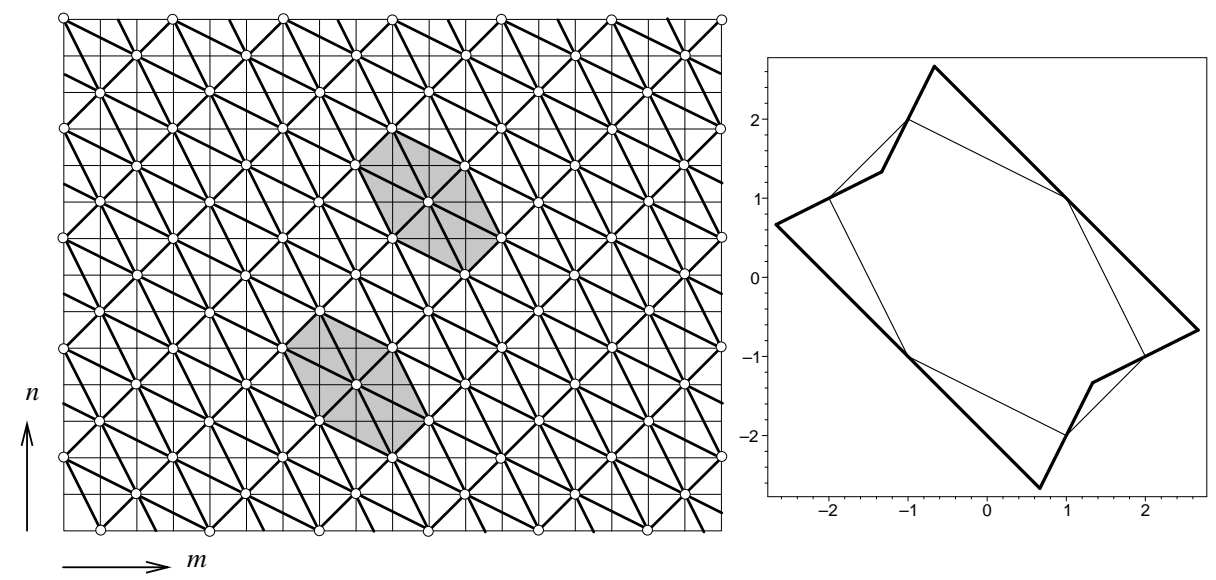

Fig. 10. Left: Pattern for producing the dual mesh from the dual surface. The vertical and horizontal lines represent the grid of asymptotic lines, while the circles indicate which points should be sampled. Right: The limit shape (thick curve) of the star defined by the thin polygon, which corresponds to the hexagons shown in the left figure.

surface at the corresponding vertex. The global polarity $\pi$, however, modifies this shape by a projective transformation which can be shown to preserve the regularity, provided that the step-size is sufficiently small

We illustrate this result by two examples.

Example 6. Consider the Enneper surface

$$
\left.\left(\frac{1}{3}(u+v)\left(2 u^{2}-8 u v+3+2 v^{2}\right), \frac{1}{3}(u-v)\left(2 u^{2}+8 u v+3+2 v^{2}\right), 4 u v+1\right)\right),
$$

where the parameter lines are already the asymptotic lines. The dual surface has the parametric representation

$$
\mathbf{P}(u, v)=\left(\begin{array}{c}
\frac{6(u+v)}{\left(8 u^{3} v+6 u^{2}+8 u v^{3}+12 u v+6 v^{2}-3\right)} \\
\frac{-6(-v+u)}{\left(8 u^{3} v+6 u^{2}+8 u v^{3}+12 u v+6 v^{2}-3\right)} \\
\frac{3\left(2 v^{2}+2 u^{2}-1\right)}{\left(8 u^{3} v+6 u^{2}+8 u v^{3}+12 u v+6 v^{2}-3\right)}
\end{array}\right) .
$$

By choosing $\alpha(t)=\mathbf{P}(t, t)$ and suitable $h$ we obtain a dual mesh of $\mathbf{P}$ and finally a regular primal mesh which approximates the Enneper surface - see Figure 11.

This example is somewhat special, since the asymptotic lines intersect each other orthogonally (as it is a minimal surface). This is not the case of the second example.

Example 7. We consider a segment of a ruled quadric, where the parameter lines are the two families of straight lines on this surface. By applying the algorithm with three different step-sizes we obtain the primal meshes shown in Fig. 12. 


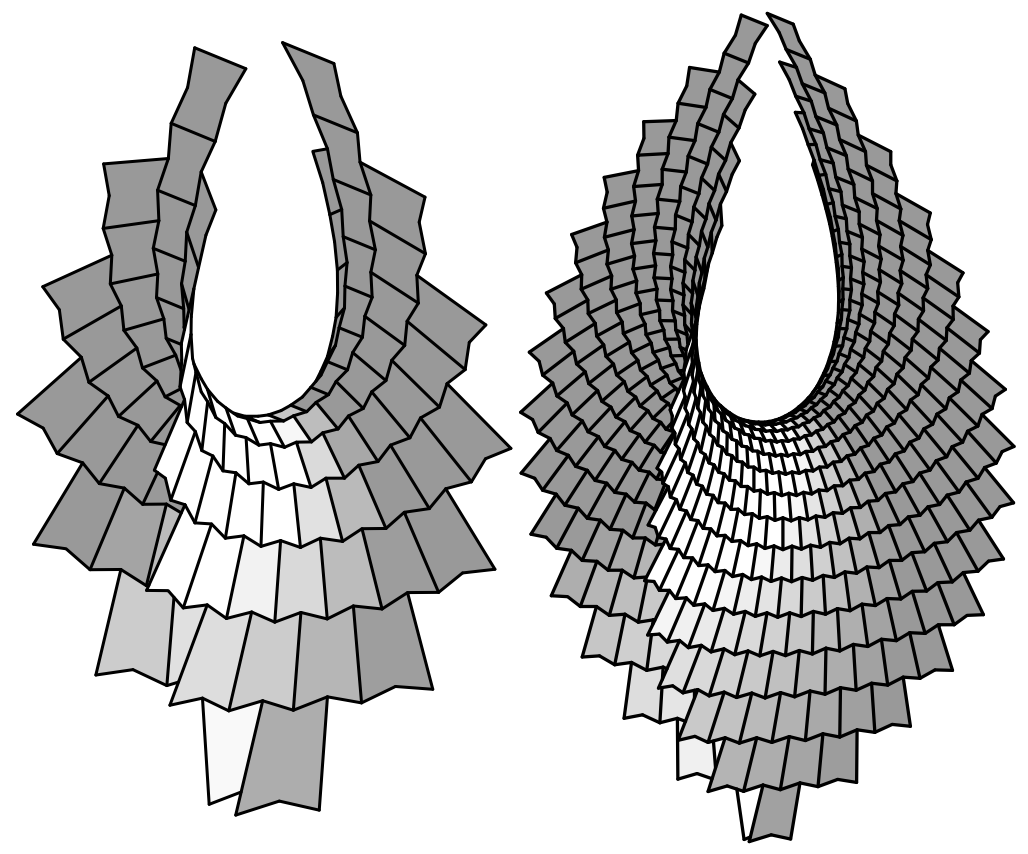

Fig. 11. Primal tangent meshes of the Enneper surface for values $h=0.081$ (left) and $h=0.039$ (right).

Clearly, this technique can also be used to analyze other patterns than the one shown in Fig. 10, including non-uniform ones. This will be a topic of future research.

\subsection{The case of elliptic surfaces}

If the given primal surface patch has only elliptic points, then a different algorithm can be used. We assume that the Gauss image of the given patch is contained in a hemisphere. In addition, it should be convex, i.e., any great circular arc connecting two points of the Gauss image should entirely belong to the Gauss image, too.

An approximation of the primal surface by a regular mesh can be obtained as follows.

\section{Algorithm 2}

1. Choose a spherical triangulation of the sphere.

2. For all vertices $\mathbf{n}_{i}, i=1, \ldots, m$, evaluate the support function of the given surface, $h_{i}=h\left(\mathbf{n}_{i}\right)$ and compute the corresponding points $\mathbf{t}_{i}=\frac{1}{h_{i}} \mathbf{n}_{i}$ of the dual mesh.

3. Compute the convex hull of the points $\left\{\mathbf{t}_{i}, i=1, \ldots, m\right\}$. 


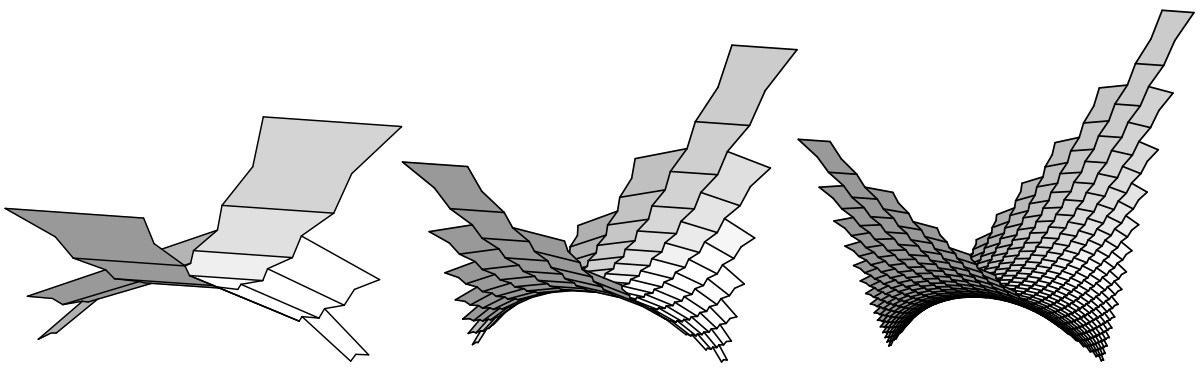

Fig. 12. Primal tangent meshes of a ruled quadric for three different values of $h$.

4. The apparent contour of the convex hull, seen from the origin, splits the boundary surface of the convex hull into two meshes. One of them is the suitable dual mesh; the other one does not have any inner vertices.

Due to the fact that the dual surface of the elliptic point is also elliptic, all vertices of the dual mesh obtained from this algorithm have convex stars. Consequently, all faces of the primal mesh are convex (and therefore simple) polygons.

An example has already been presented in Section 4.5 (Example 3).

\section{Conclusion}

Based on the use of piecewise linear support functions we discussed dual meshes, which were assumed to be star-shaped with respect to the origin, and the associated primal meshes. The primal meshes are capable of approximating smooth surface patches without parabolic points. It should be noted that these meshes approximate not only the surfaces but also the associated normals. I.e., each normal in the Gauss image corresponds to exactly one normal on the primal mesh. This is clearly not the case for general meshes which approximate a given surface.

As a matter of future work we will use the support function for generating error bounds. In the smooth case the maximum distance error is essentially equal to the maximum difference between the original support function and its approximation. While this is also true for convex primal meshes, the extension of this result to the non-convex case is still an open problem.

In addition, we plan to discuss the approximation of general support functions by piecewise linear support functions over a given spherical triangulation, subject to conditions which guarantee the regularity of the resulting primal mesh. In the case of elliptic surfaces, this can be formulated as an optimization problem with linear constraints. In the hyperbolic case, however, non-linear constraints are needed.

Finally we plan to investigate surfaces with parabolic lines separating elliptic and hyperbolic regions. In order to represent these surfaces, multi-valued piecewise linear functions will be needed. 
Acknowledgment. This research was supported by a grant of the Austrian Science Fund (FWF, project no. P17387-N12 and SFB F013, subproject 15), by research project no. MSM 0021620839 at Charles University, Prague, and by the project "Facetted glass shells" at the Department of Civil Engineering of Danish Technical University. The authors wish to thank the reviewers for their useful comments. Special thanks go to Bert's father for building the two models shown in Figures 7 and 8.

\section{References}

1. H. Almegaard: The stringer system - a truss model of membrane shells for analysis and design of boundary conditions. International Journal of Space Structures $\mathbf{1 9}$ (2004), 1-10.

2. T. Bonnesen and W. Fenchel: Theory of convex bodies. BCS Associates, Moscow, Idaho, 1987.

3. M. Brückner: Vielecke und Vielflache - Theorie und Geschichte, Teubner, Leipzig 1900.

4. B. Cutler and E. Whiting: Constrained Planar Remeshing for Architecture, in: Symposium on Geometry Processing 2006, Poster proceedings (electronic), p. 5, http://sgp2006.sc.unica.it/program/PosterProceedings.pdf.

5. H. Groemer: Geometric Applications of Fourier Series and Spherical Harmonics Cambridge University Press, Cambridge, 1996.

6. P. M. Gruber and J. M. Wills (eds.): Handbook of convex geometry, North-Holland, Amsterdam, 1993.

7. J. Hoschek and D. Lasser: Fundamentals of Computer Aided Geometric Design, AK Peters, Wellesley Mass., 1996.

8. J. Hoschek: Dual Bézier curves and surfaces, in: Surfaces in Computer Aided Geometric Design, R.E. Barnhill and W. Boehm, eds., North Holland, 1983, 147-156.

9. H. Kawarahada and K. Sugihara: Dual subdivision: A new class of subdivision schemes using projective duality, in WSCG'2006 Full paper proceedings, J. Jorge and V. Skala, eds., University of West Bohemia, Plzen 2006, 9-16.

10. Y. Liu, H. Pottmann, J. Wallner, Y. Yang and W. Wang: Geometric Modeling with Conical Meshes and Developable Surfaces. ACM Trans. Graphics 25 (Siggraph 2006), 681-689.

11. G. Patanè and M. Spagnuolo: Triangle Mesh Duality: Reconstruction and Smoothing, in The Mathematics of Surfaces X, M. Wilson and R. Martin, eds., Springer LNCS 2768, 111-128, Berlin 2003.

12. H. Pottmann and J. Wallner, The focal geometry of circular and conical meshes, Adv. Comput. Math., to appear.

13. L. Ros, K. Sugihara, F. Thomas: Towards shape representation using trihedral mesh projections, The Visual Computer 19 (2003), 139-150.

14. M. Sabin: A Class of Surfaces Closed under Five Important Geometric Operations, Technical report no. VTO/MS/207, British aircraft corporation, 1974, available at http://www.damtp.cam.ac.uk/user/na/people/Malcolm/vtoms/vtos.html

15. Z. Sír, J. Gravesen and B. Jüttler: Curves and surfaces represented by polynomial support functions, SFB report no. 2006-36, available at http://www.sfb013. uni-linz.ac.at.

16. E. W. Weisstein: Dual Polyhedron. From MathWorld - A Wolfram Web Resource. http://mathworld. wolfram.com/DualPolyhedron.html

17. M. J. Wenninger: Dual models, Cambridge University Press 1983. 